Western University

Scholarship@Western

Medical Biophysics Publications

Medical Biophysics Department

3-10-2015

Investigation of the best model to characterize diffuse correlation spectroscopy measurements acquired directly on the brain

KVerdecchia

Western University

M Diop

Western University

KSt. Lawrence

Western University

Follow this and additional works at: https://ir.lib.uwo.ca/biophysicspub

Part of the Medical Biophysics Commons

Citation of this paper:

Verdecchia, K; Diop, M; and St. Lawrence, K, "Investigation of the best model to characterize diffuse correlation spectroscopy measurements acquired directly on the brain" (2015). Medical Biophysics Publications. 63.

https://ir.lib.uwo.ca/biophysicspub/63 


\title{
Investigation of the best model to characterize diffuse correlation spectroscopy measurements acquired directly on the brain
}

\author{
K Verdecchia ${ }^{1,2}$, M Diop ${ }^{1,2}$, and K St. Lawrence ${ }^{1,2}$
}

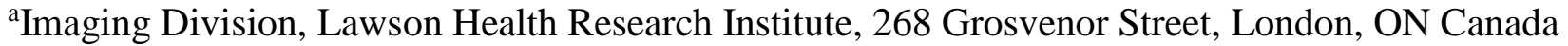

N6A 4V2; ${ }^{b}$ Department of Medical Biophysics, Western University, 1151 Richmond Street North, London, ON Canada N6A 5C1

\begin{abstract}
Diffuse correlation spectroscopy (DCS) is a non-invasive optical technique capable of monitoring tissue perfusion changes, particularly in the brain. The normalized temporal intensity autocorrelation function generated by DCS is typically characterized by assuming that the movement of erythrocytes can be modeled as a Brownian diffusion-like process instead of the expected random flow model. Carp et al. [Biomedical Optics Express, 2011] proposed a hybrid model, referred to as the hydrodynamic diffusion model, to capture both the random ballistic and diffusive nature of erythrocyte motion. The purpose of this study was to compare how well the Brownian diffusion and the hydrodynamic diffusion models characterized DCS data acquired directly on the brain, avoiding the confounding effects of scalp and skull. Data were acquired from seven pigs during normocapnia $(39.9 \pm 0.7 \mathrm{mmHg})$ and hypocapnia $(22.1 \pm 1.6 \mathrm{mmHg})$ with the DCS fibers placed $7 \mathrm{~mm}$ apart, directly on the cerebral cortex. The hydrodynamic diffusion model was found to provide a consistently better fit to the autocorrelation functions compared to the Brownian diffusion model and was less sensitive to the chosen start and end time points used in the fitting. However, the decrease in cerebral blood flow from normocapnia to hypocapnia determined was similar for the two models $(-42.6 \pm 8.6 \%$ for the Brownian model and $42.2 \pm 10.2 \%$ for the hydrodynamic model), suggesting that the latter is reasonable for monitoring flow changes.
\end{abstract}

Keywords: diffuse correlation spectroscopy, Brownian, hydrodynamic, cerebral cortex, juvenile pig, blood flow

\section{INTRODUCTION}

Near-infrared spectroscopy (NIRS) is an optical technique capable of measuring various physiological parameters, such as oxygenation, blood volume, and blood flow ${ }^{1}{ }^{3}$. Many NIRS modalities determine these physiological parameters by measuring the light absorption caused by chromophores, such as deoxy- and oxy-hemoglobin, within the tissue that the light has propagated. To measure blood flow using the NIRS light absorption method, injection of a bolus of contrast agent is required and the light absorption caused by the contrast agent is measured during the passage of the bolus ${ }^{4,5}$. Another approach is diffuse correlation spectroscopy (DCS) that measures relative blood flow changes by monitoring light intensity changes caused by the motion of scatters, which in tissue is dominated by the movement of erythrocytes.

The most common approach for analyzing DCS data is to model erythrocyte movement as a Brownian diffusion-like process. This model has been shown to provide better characterization of normalized temporal intensity autocorrelation functions obtained from various species (swine, rodent and human) than the expected random flow model ${ }^{6}-9$. To explain this unexpected finding, Carp et al. proposed a hybrid model, referred to as the hydrodynamic diffusion model that accounts for the possibility of multiple light-scattering events within a vessel, which would be characteristic of diffusive motion, and light-scattering events across vessels, which is characteristic of random ballistic motion ${ }^{10}$. The purpose of the current study was to compare the ability of these different flow models to characterize DCS data acquired directly on the exposed cerebral cortex of juvenile pigs. This approach avoids complications due to tissue heterogeneity, which can also alter the shape of DCS curves due to the different flow rates in intra- and extra-cerebral tissue ${ }^{11}$.

\subsection{Diffuse Correlation Spectroscopy}

To assess the scattering dynamics of detected light, DCS measures the normalized intensity autocorrelation function, $g_{2}(\rho, \tau)$, given by:

$$
g_{2}(\rho, \tau) \propto \frac{\langle I(\rho, t) I(\rho, t+\tau)\rangle}{\langle I(\rho, t)\rangle^{2}}
$$

Biomedical Applications of Light Scattering IX, edited by Adam Wax, Vadim Backman, Proc. of SPIE Vol. 9333, 93330E · @ 2015 SPIE · CCC code: 1605-7422/15/\$18 · doi: 10.1117/12.2079499 
Equation (1) is related to the electric field autocorrelation function by the Siegert relation ${ }^{12}$ :

$$
\mathrm{g}_{2}(\rho, \tau)=1+\frac{\beta\left|\mathrm{G}_{1}(\rho, \tau)\right|^{2}}{\langle\mathrm{I}(\rho, \mathrm{t})\rangle^{2}}
$$

where, $\beta$ is the coherence factor of the detection channel and $G_{1}(\rho, \tau)$ is the analytical solution to the diffusion equation and for a semi-infinite homogenous medium, such as brain tissue, is given by:

$$
G_{1}(\rho, \tau)=\frac{3 \mu_{s}^{\prime}}{4 \pi}\left(\frac{\exp \left(-k_{D} r_{1}\right)}{r_{1}}-\frac{\exp \left(-k_{D} r_{2}\right)}{r_{2}}\right)
$$

Where, $r_{1}=\sqrt{\rho^{2}+z_{0}^{2}}, r_{2}=\sqrt{\rho^{2}+\left(z_{0}+2 z_{b}\right)^{2}}, \rho$ is the source-detector distance (SDD), $z_{b}$ is the distance above the tissue surface at which the fluence vanishes, and $z_{0}$ is the effective depth of the source $e^{4,13}$.

Also:

$$
\mathrm{k}_{\mathrm{D}}^{2}=3 \mu_{\mathrm{a}} \mu_{\mathrm{s}}^{\prime}+\mu_{\mathrm{s}}^{\prime 2} \mathrm{k}_{0}^{2} \alpha\left\langle\Delta \mathrm{r}^{2}(\tau)\right\rangle
$$

Where $\mu_{\mathrm{a}}$ and $\mu_{\mathrm{s}}^{\prime}$ are the absorption and reduced scattering coefficient, respectively, $\mathrm{k}_{0}$ is the wavenumber of light in the medium, $\alpha$ is the proportion of moving scatters (predominantly red blood cells) to all scatterers (i.e., related to the blood volume) and $\left\langle\Delta \mathrm{r}^{2}(\tau)\right\rangle$ is the mean-square displacement of the moving scatterers during a correlation time $\tau$. Therefore, the measured $g_{2}(\rho, \tau)$ curves were fit with the analytical model to extract $\alpha\left\langle\Delta \mathrm{r}^{2}(\tau)\right\rangle$ by using either measured or assumed values for the optical properties $\left(\mu_{\mathrm{a}}\right.$ and $\left.\mu_{\mathrm{s}}^{\prime}\right)$ and one of the two flow models to describe the displacement of red blood cells.

\subsection{Brownian Diffusion Model}

The dominant flow model used to analyze DCS data is the simplified Brownian displacement formula, which is given by:

$$
\left\langle\Delta \mathrm{r}^{2}(\tau)\right\rangle=6 \mathrm{D}_{\mathrm{B}} \tau
$$

where, $D_{B}$ is the effective Brownian diffusion coefficient. This model assumes many flow-related scattering events occur quickly, such that movement of red blood cells behaves as a random walk process. The two fitting parameters are $\beta$ and $\alpha D_{B}$, where the latter is referred to as the Brownian blood flow index $\left(\mathrm{BFI}_{\mathrm{B}}\right)$.

\subsection{Hydrodynamic Diffusion Model}

Carp et al. proposed using the Langevin formula for red blood cell mean squared displacement to account for the occurrence of both Brownian and random flow motion ${ }^{10}$ :

$$
\left\langle\Delta \mathrm{r}^{2}(\tau)\right\rangle=6 \mathrm{D}_{\mathrm{H}}\left\{\tau-\tau_{\mathrm{c}}\left[1-\exp \left(-\frac{\tau}{\tau_{\mathrm{c}}}\right)\right]\right\}
$$

where $D_{H}$ is the effective hydrodynamic diffusion coefficient and $\tau_{c}$ is the time scale required to establish diffusive motion. Unlike the Brownian diffusion model, the displacement term $\left(\tau_{\mathrm{c}}\right)$ from Equation (6) describes the ballistic motion at short delay times. For the hydrodynamic diffusion model $\left(\mathrm{H}_{\mathrm{DM}}\right)$, the three parameters are $\beta, \tau_{\mathrm{c}}$ and $\alpha \mathrm{D}_{\mathrm{H}}$, where the latter is referred to as the hydrodynamic blood flow index $\left(\mathrm{BFI}_{\mathrm{H}}\right)$.

\section{METHODS}

\subsection{Diffuse Correlation Spectroscopy System}

The DCS system used a continuous-wave laser (DL785-100-S, CrystalLaser, Nevada) emitting at $785 \mathrm{~nm}$ with a maximum output power of $100 \mathrm{~mW}$ and a coherence length $>5 \mathrm{~m}$. The emitted light was attenuated with an electronically controlled variable neutral density filter (NDC-50-4M, Thorlabs) and coupled into a multimode emission fiber (emission probe: N.A. $=0.22$, core $=400 \mu \mathrm{m}, 4.7$-mm outer diameter; Fiberoptics Technology, Pomfret, CT). A fiber bundle was used for detecting the temporal light intensity fluctuations. The fiber bundle had a common end (length $=1.5 \mathrm{~m}$ ), which was placed on the subject, and branched into four single-mode fibers (SMF-28e+, N.A. $=0.14$, length $=$ $2.5 \mathrm{~m}$, core $=8.2 \mu \mathrm{m}$, single-mode cutoff wavelength at $1260 \mathrm{~nm}$ ) that connected to four individual input-channels for DCS detection; however, only a single input-channel was required for this study due to the sufficient count rate per channel $(>200 \mathrm{kHz})$. The DCS detection fibers were wrapped into a 5-cm coil to increase the losses in the higher-order 
modes before being coupled to their respective avalanche photodiode of a single photon counting module (SPCMAQ4C, Excelitas Canada Inc, QC, Canada). The output of the SPCM was sent to a photon correlator board (DPC-230, Beker \& Hickl, Germany) that computed the normalized intensity autocorrelation functions $\left(\mathrm{g}_{2}\right)$.

\subsection{Experimental Procedure}

Animal experiments were conducted according to the guidelines of the Canadian Council of Animal Care (CCAC) and approved by the Animal Use Committee at Western University. Seven juvenile pigs ( 2 weeks old, weight $=16.0 \pm 3.2$ $\mathrm{kg}, 6$ females, 1 male) were obtained from a local supplier on the day of the experiment. Following anesthetic induction with $1.75-3 \%$ isoflurane, the animals were tracheotomized and mechanically ventilated on a mixture of oxygen and medical air. A catheter was inserted into a femoral artery to draw blood for analysis of oxygen and carbon dioxide levels with a portable blood gas analyzer (Radiometer, ABL 80 FLEX CO-OX, Denmark) throughout the experiment. To expose the brain, the extracerebral layers were removed. First, a rectangular piece of scalp tissue $\left(\sim 10 \mathrm{~cm}^{2}\right)$ was removed from the outermost cerebral layer located lateral to the midline on the right hemisphere. Next, caustic pencils containing silver nitrate were used to cauterize the incised scalp tissue. Finally, two burr holes, the size of an optical fiber, were drilled with a handheld dremel into the skull $\sim 7 \mathrm{~mm}$ apart, with the exception that animal six was drilled $\sim 20$ $\mathrm{mm}$ apart. Care was taken to not puncture the dura mater with the dremel when exposing the brain. Before DCS data were collected, a blood sample from the femoral artery was analyzed to verify that the animal was at normocapnia (38$42 \mathrm{mmHg}$ ). Next, the DCS data were acquired for 90 seconds, followed by the analysis of a second blood sample to demonstrate no significant change in arterial blood $\mathrm{CO}_{2}$ tension. Finally, cerebral blood flow was reduced by inducing hypocapnia (20-25 $\mathrm{mmHg}$ ), which was achieved by increasing the respiration rate. DCS data were acquired following the same procedure as under normocapnia.

\subsection{Data Analysis}

Four variations of the fitting procedure were used to analyze the autocorrelation data: the two flow models $\left(\mathrm{B}_{\mathrm{DM}}\right.$ and $\left.\mathrm{H}_{\mathrm{DM}}\right)$ and two fitting ranges. In all cases the fitting started at a correlation time $\left(\tau_{1}\right)$ of $0.3 \mu \mathrm{s}$; however, the largest correlation time $\left(\tau_{2}\right)$ was either limited to the value defined by $g_{1}=0.7$, to restrict the fitting to early correlation times, or to a value corresponding to $g_{1}=0.01$ to include the entire autocorrelation curve. The former fitting range is referred to as the "early" fitting technique, which is known to have greater sensitivity to photons that travelled into deeper tissue, and the latter will be referred to as the "late" fitting technique. The average $\tau_{2}$ values for the "early" fitting technique were $\tau_{2}=7.8 \pm 2.6$ and $12.6 \pm 3.4 \mu$ s during normocapnia and hypocapnia, respectively, and $\tau_{2}=1 \mathrm{~ms}$ for the "late" fitting technique.

To determine the better fitting model, an F-test was used to account for the differences in the number of fitting parameters for the two models (two for $\mathrm{B}_{\mathrm{DM}}$ and three for $\mathrm{H}_{\mathrm{DM}}$ ). Since the correlation time fitting threshold varied for the "early" fitting techniques, the number of degrees of freedom used in the F-test was determined by the number of correlation bins that the individual fit required. For the $\mathrm{H}_{\mathrm{DM}}$ to better characterize the data compared to the $\mathrm{B}_{\mathrm{DM}}$, the calculated F-number had to exceed a critical F-value of 4, as determined by the finv function in MATLAB (Mathworks, Natick, MA) for a $\mathrm{p}<0.05$.

\section{RESULTS AND DISCUSSION}

\subsection{Physiological Parameters}

A total of seven juvenile pigs (six females, one male) were used in the study and all errors are given as standard deviations. The average weight of the animals was $16.0 \pm 3.2 \mathrm{~kg}$. 
Table 1. Average physiological parameters of the seven animals.

\begin{tabular}{|c|c|c|c|c|c|c|}
\hline Condition & $\mathbf{p H}$ & $\begin{array}{c}\mathbf{p C O} \\
{[\mathbf{m m H g}]}\end{array}$ & $\begin{array}{c}\mathbf{p O} \\
{[\mathbf{m m H g}]}\end{array}$ & $\begin{array}{c}\mathbf{c t H b} \\
{[\mathbf{g} / \mathbf{d L}]}\end{array}$ & $\begin{array}{c}\mathbf{M A P} \\
{[\mathbf{m m H g}]}\end{array}$ & $\begin{array}{c}\text { HR } \\
{[\mathbf{b p m}]}\end{array}$ \\
\hline Normocapnia & $7.44 \pm 0.06$ & $39.9 \pm 0.7$ & $165 \pm 26$ & $10.3 \pm 0.8$ & $44.6 \pm 7.2$ & $131 \pm 27$ \\
\hline Hypocapnia & $7.60 \pm 0.07$ & $22.1 \pm 1.6$ & $197 \pm 41$ & $9.8 \pm 1.3$ & $40.5 \pm 5.5$ & $128 \pm 27$ \\
\hline
\end{tabular}

Table 1 lists the physiological parameters measured during normocapnia and hypocapnia, where $\mathrm{pCO}_{2}, \mathrm{pO}_{2}, \mathrm{ctHb}, \mathrm{MAP}$, and HR represent the partial carbon dioxide tension, partial oxygen tension, total hemoglobin concentration, mean arterial pressure, and heart rate. Significant differences were observed between the two conditions for $\mathrm{pH}^{\mathrm{and}} \mathrm{pCO}_{2}$ $(\mathrm{p}<0.05)$. Other physiological parameters, such as blood glucose concentration, temperature and arterial saturation, did not change significantly between the two conditions; their mean values were $4.0 \pm 1.2 \mathrm{mmol} / \mathrm{L}, 38.3 \pm 0.5^{\circ} \mathrm{C}$, and $95.1 \pm$ $5.9 \%$, respectively.

\subsection{Example of the Best-Fit of the two Flow Models}

Figure 1 shows an example of autocorrelation decay curves obtained on the exposed cerebral cortex of an animal under normocapnia and hypocapnia conditions.

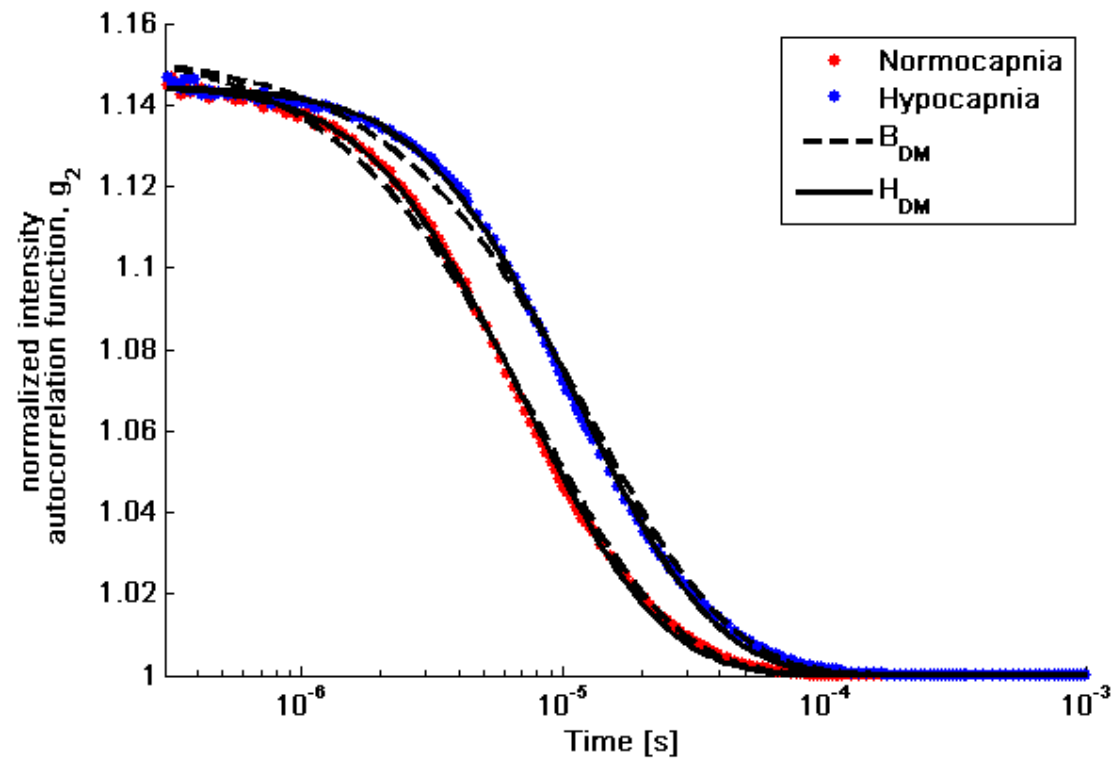

Figure 1. A sample of DCS autocorrelation curves obtained during normocapnia (red) and hypocapnia (blue). Also shown are best-fits of $\mathrm{B}_{\mathrm{DM}}$ (dashed line) and $\mathrm{H}_{\mathrm{DM}}$ (solid line) measured with a count rate of $\sim 750 \mathrm{kHz}$.

As anticipated, the normalized intensity autocorrelation function under normocapnia decayed faster than under hypocapnia due to the faster blood flow under the former condition. In this specific example, the percent blood flow change from normocapnia to hypocapnia was $-39 \%$ and $-37 \%$ measured by the $\mathrm{B}_{\mathrm{DM}}$ and the $\mathrm{H}_{\mathrm{DM}}$, respectively. Figure 1 illustrates the poorer fit by the $\mathrm{B}_{\mathrm{DM}}$ compared to the $\mathrm{H}_{\mathrm{DM}}$, especially during the early correlation times $\left(<10^{-5} \mathrm{~s}\right)$.

\subsection{Change in Blood Flow}

The average BFI extracted from the four fitting variations are presented in Figure 2. 


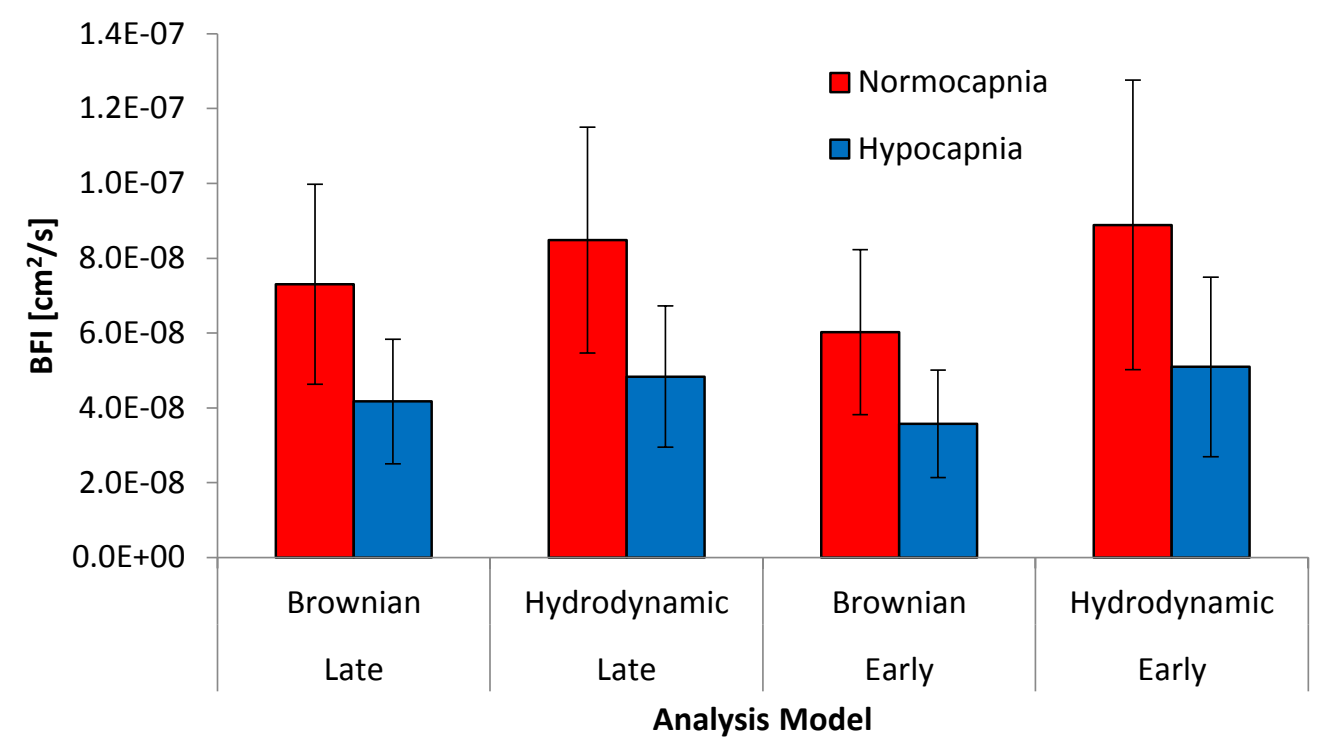

Figure 2. Average BFI extracted by the four fitting methods for the seven animals plotted for normocapnia (red) and hypocapnia (blue) conditions. The "early" and "late" techniques were defined by $\tau_{2}$ at the threshold of $\mathrm{g}_{1}<0.7$ and $\mathrm{g}_{1}<0.01$, respectively.

Figure 2 shows that for all fitting techniques the extracted BFI, was significantly different $(p<0.05)$ between normocapnia and hypocapnia with no significant difference observed between fitting techniques. The similarity between the two fitting endpoint techniques, "early" and "late", demonstrates that the semi-infinite homogenous model assumption is valid for the exposed cerebral cortex, as expected for a global change in CBF such as caused by manipulating arterial carbon dioxide tension.

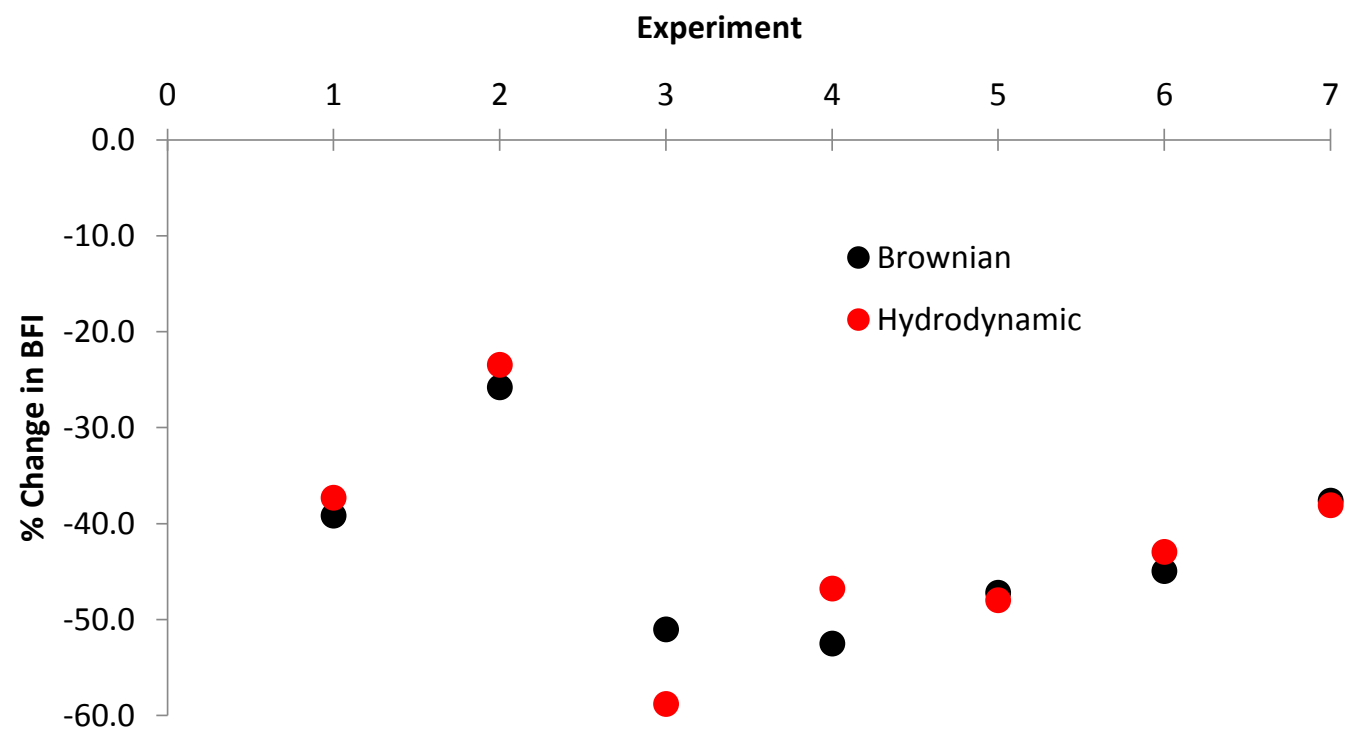

Figure 3. The percent change in the BFI measured from individual animals. Each study was fit using the four fitting variations.

Figure 3 shows that fitting the data with either model resulted in similar changes in cerebral blood flow for each animal. The greatest variation occurred in the third study $(9.7 \%)$ and the average coefficient of variance across all seven studies was $5.5 \pm 2.5 \%$. 
Table 2. Average BFI and $\beta$ values measured during normocapnia and hypocapnia by the two flow models.

\begin{tabular}{|c|c|c|c|c|c|c|}
\cline { 2 - 7 } \multicolumn{1}{c|}{} & \multicolumn{3}{c|}{ Normocapnia } & \multicolumn{3}{c|}{ Hypocapnia } \\
\hline Model & $\mathbf{B F I}\left[\mathbf{c m}^{\mathbf{2}} / \mathbf{s}\right] \mathbf{x} \mathbf{1 0}^{\mathbf{8}}$ & $\boldsymbol{\beta}$ & $\boldsymbol{\tau}_{\mathbf{c}}[\boldsymbol{\mu s}]$ & $\mathbf{B F I}\left[\mathbf{c m}^{2} / \mathbf{s}\right] \mathbf{x ~ 1 0}$ & $\boldsymbol{\beta}$ & $\boldsymbol{\tau}_{\mathbf{c}}[\boldsymbol{\mu s}]$ \\
\hline $\mathrm{B}_{\mathrm{DM}}$ & $7.31 \pm 2.68$ & $0.166 \pm 0.011$ & --- & $4.17 \pm 1.66$ & $0.162 \pm 0.01$ & -- \\
\hline $\mathrm{H}_{\mathrm{DM}}$ & $8.49 \pm 3.02$ & $0.158 \pm 0.012$ & $1.57 \pm 1.33$ & $4.83 \pm 1.89$ & $0.154 \pm 0.01$ & $2.63 \pm 0.93$ \\
\hline
\end{tabular}

The $\beta$ values in Table 2 are close to the theoretical value of 0.167 for the few-mode fiber utilized in this study. Between conditions (normocapnia and hypocapnia), the BFI was not significant between models and, as expected, the coherence factor $\beta$ was not significant. Also, the third parameter for the $\mathrm{H}_{\mathrm{DM}}\left(\tau_{\mathrm{c}}\right)$ did not change significantly between conditions; however, appeared the observed trend suggests that significance may be reached with a larger sample size.

Table 3. Average change in BFI and $\beta$ measured for the two flow models.

\begin{tabular}{|c|c|c|}
\hline Model & BFI [\%] & $\boldsymbol{\beta}[\%]$ \\
\hline $\mathrm{B}_{\mathrm{DM}}$ & $-42.6 \pm 9.3$ & $-2.3 \pm 1.6$ \\
\hline $\mathrm{H}_{\mathrm{DM}}$ & $-42.2 \pm 11.0$ & $-2.3 \pm 3.0$ \\
\hline
\end{tabular}

Table 3 shows the good agreement in the percent BFI change between the two models. The "early" fitting technique improved the $\beta$ percent difference between conditions to $<1.6 \%$, maintaining similar BFI $\%$ differences $(-41 \pm 7.9$ and $42 \pm 9.9 \%, \mathrm{~B}_{\mathrm{DM}}$ and $\left.\mathrm{B}_{\mathrm{DM}}\right)$.

\subsection{Better Fitting Model}

A simple comparison of the sum of squares obtained by both flow models.

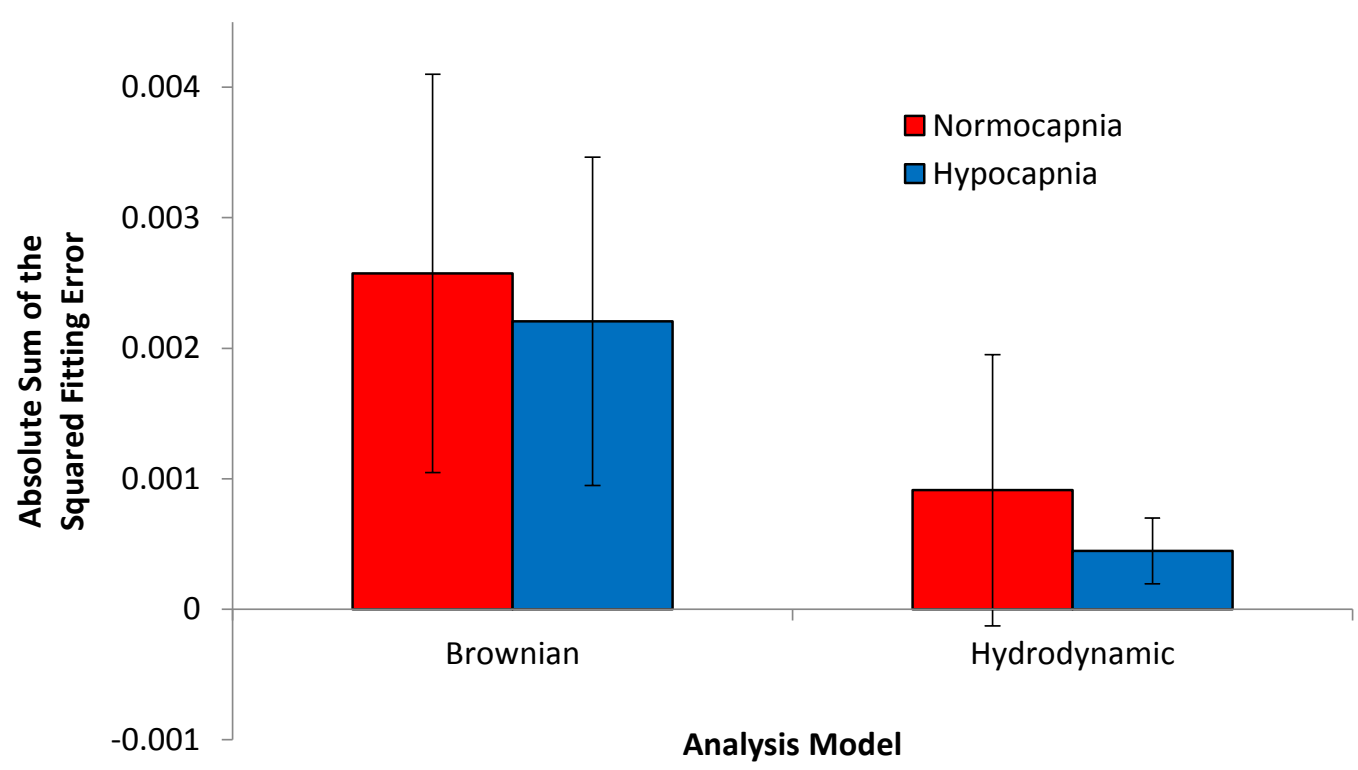

Figure 4. Sum of the squared fitting error calculated from the two flow models during each condition (normocapnia and hypocapnia shown in red and blue, respectively).

Figure 4 indicates that the hydrodynamic flow model provided a bettering fit than the Brownian flow model, which is not unexpected considering the $\mathrm{H}_{\mathrm{DM}}$ includes an additional fitting parameter. Therefore, an F-test, as described in the methods, was used to determine the better fitting model. For the seven animals in this study, five showed a significant difference between flow models during normocapnia and all seven showed that the $\mathrm{H}_{\mathrm{DM}}$ significantly fit better than the B $\mathrm{DM}$. 


\section{CONCLUSION}

In this work, the ability of two flow models, $\mathrm{B}_{\mathrm{DM}}$ and the $\mathrm{H}_{\mathrm{DM}}$, to quantify perfusion changes measured directly on the brain of juvenile pigs was investigated. As expected for a semi-infinite homogenous model, no differences were observed in perfusion changes obtained by fitting the entire decay curve and fitting only the earlier portion of the curve, which is more sensitive to deeper tissues. This was expected because exposing the brain removed signal contamination from extra-cerebral tissue and the region of the brain interrogated by the light was relatively homogenous with respect to blood flow (i.e. predominately cerebral cortex). The most interesting finding from this study was that, although the $\mathrm{H}_{\mathrm{DM}}$ fit the data better, there were no significant differences observed between the cerebral cortex blood flow changes measured by the two models. In agreement with Carp et al, accounting for the random ballistic flow by erythrocytes with the $\mathrm{H}_{\mathrm{DM}}$ improved the characterization of the autocorrelation data, especially during early correlation times $\left(<10^{-5}\right.$ $\mathrm{s})$, but the effects from the diffusion flow of erythrocytes predominates; therefore, the $\mathrm{B}_{\mathrm{DM}}$ is reasonable for monitoring flow changes.

\section{ACKNOWLEDGMENTS}

This work was supported by grants from the Canadian Institutes of Health Research, and the National Science and Engineering Council of Canada and a personnel award to K. St. Lawrence from the Heart and Stroke Foundation, Ontario Provincial Office. K. Verdecchia was supported by a scholarship from an Ontario Graduate Scholarship.

\section{REFERENCES}

[1] Tichauer, K.M., Elliott, J.T., Hadway, J., Lee, T.-Y., and St. Lawrence, K., "Cerebral metabolic rate of oxygen and amplitude-integrated electroencephalography during early reperfusion after hypoxia-ischemia in piglets.," Journal of applied physiology 106(5), 1506-12 (2009).

[2] Verdecchia, K., Diop, M., Lee, T.-Y., and Lawrence, K. St., "Quantifying the cerebral metabolic rate of oxygen by combining diffuse correlation spectroscopy and time-resolved near-infrared spectroscopy," Journal of biomedical optics 18(2), (2013).

[3] Elliott, J.T., Diop, M., Morrison, L.B., d'Esterre, C.D., Lee, T.-Y., and St. Lawrence, K., "Quantifying cerebral blood flow in an adult pig ischemia model by a depth-resolved dynamic contrast-enhanced optical method.," NeuroImage 94C, 303-311 (2014).

[4] Diop, M., Verdecchia, K., Lee, T.-Y., and St. Lawrence, K., "Calibration of diffuse correlation spectroscopy with a time-resolved near-infrared technique to yield absolute cerebral blood flow measurements.," Biomedical optics express 2(7), 2068-81 (2011).

[5] Brown, D.W., Picot, P. a, Naeini, J.G., Springett, R., Delpy, D.T., and Lee, T.-Y., "Quantitative near infrared spectroscopy measurement of cerebral hemodynamics in newborn piglets.," Pediatric research 51(5), 564-70 (2002).

[6] Zhou, C., Eucker, S. a, Durduran, T., Yu, G., Ralston, J., Friess, S.H., Ichord, R.N., Margulies, S.S., and Yodh, A.G., "Diffuse optical monitoring of hemodynamic changes in piglet brain with closed head injury.," Journal of biomedical optics 14(3), 034015 (2009).

[7] Kim, M.N., Durduran, T., Frangos, S., Edlow, B.L., Buckley, E.M., Moss, H.E., Zhou, C., Yu, G., Choe, R., et al., "Noninvasive measurement of cerebral blood flow and blood oxygenation using near-infrared and diffuse correlation spectroscopies in critically brain-injured adults.," Neurocritical care 12(2), 173-80 (2010).

[8] Mesquita, R.C., Durduran, T., Yu, G., Buckley, E.M., Kim, M.N., Zhou, C., Choe, R., Sunar, U., and Yodh, A.G., "Direct measurement of tissue blood flow and metabolism with diffuse optics.," Philosophical transactions. Series A, Mathematical, physical, and engineering sciences 369(1955), 4390-406 (2011).

[9] Yu, G., Floyd, T.F., Durduran, T., Zhou, C., Wang, J., Detre, J. a, and Yodh, A.G., "Validation of diffuse correlation spectroscopy for muscle blood flow with concurrent arterial spin labeled perfusion MRI.," Optics express 15(3), 1064-75 (2007).

[10] Carp, S.A., Roche-Labarbe, N., Franceschini, M.A., Srinivasan, V.J., Sakadžić, S., and Boas, D., "Due to intravascular multiple sequential scattering, Diffuse Correlation Spectroscopy of tissue primarily measures relative red blood cell motion within vessels.," Biomedical optics express 2(7), 2047-54 (2011). 
[11] Gagnon, L., Desjardins, M. 'R., Jehanne-Lacasse, J., Bherer, L., and Lesage, F.Ú.Ú., "Investigation of diffuse correlation spectroscopy in multi-layered media including the human head," Optics express 16(20), 1551415530 (2008).

[12] Lemieux, P.-A., and Durian, D.J., "Investigating non-Gaussian scattering processes by using nth-order intensity correlation functions," in J. Opt. Soc. Am. A 16, p. 1651 (1999).

[13] Durduran, T., Choe, R., Baker, W.B., and Yodh, A.G., "Diffuse optics for tissue monitoring and tomography," Reports on Progress in Physics 73(7), 076701 (2010).

[14] Dietsche, G., Ninck, M., Ortolf, C., Li, J., Jaillon, F., and Gisler, T., "Fiber-based multispeckle detection for time-resolved diffusing-wave spectroscopy: characterization and application to blood flow detection in deep tissue.," Applied optics 46(35), 8506-14 (2007). 